\title{
Analysis of false positivity of FDG PET-CT: Thoracic Surgery Experience
}

\author{
Fazli Yanik* \\ Trakya University School of Medicine, Thoracic Surgery Department, Edirne, Turkey
}

*Corresponding author: Fazli Yanik, Trakya University School of Medicine, Thoracic Surgery Department, Edirne, Turkey.

Received Date: February 24, 2019

Published Date: March 06, 2019

\section{Abstract}

Introduction: PET/CT (Positron Emission Tomography) is one of the current methods for tumor detection. PET/CT provides a very effective biological point of view on oncologic imaging. There are hundreds of studies that demonstrate the efficacy of PET/ CT in terms of both diagnosis and treatment planning for thoracic lesions. The aim of this study was to analysis surgically verified the false positivity of FDG PET-CT in our clinic between February 2014 and February 2019.

Material and Methods: Fifty-six patients with PET-CT positive results and preoperatively suspicious diagnosed as malignancy, but whose final histopathological results were reported as benign diseases were included in the study. Patients who had PET/CT due to intrathoracic mass and whose histopathological validation could be reached were included in the study.

Result: Twenty of the patients (34\%) were female and 36 of them (66\%) male with the mean age 54,7 $\pm 4,03$ (range 30-69) years. Wedge resection by posterolateral thoracotomy in $32(57 \%)$ patients, intraparenchymal punch biopsy by lateral thoracotomy in 10 (18\%) patients, wedge resection by VATS in $8(14 \%)$ patients, and intraparenchymal punch biopsy with VATS in $6(11 \%)$ patients was performed to reach a definitive diagnosis. No intraoperative or postoperative complications were observed in any patient. Most frequently detected false positive result was due to tuberculosis in 21 patients and the mean SUVmax value was 5,1 (range 2,5-14). Other false positive results were sarcoidosis, inflammation (pneumonia, abscess, B0OP etc.), intrapanimal lymph node, ruptured hydatid cyst, hamartoma and lastly granulomatosis with polyangiitis by frequency order. When the PET/CT values of all patients were examined, the mean SUV max value in all of the false positive cases was measured as 8,4 (0.6-26).

Conclusion: The use of PET/CT for thoracic lesions has been increasing over the last years. In addition, the detection of false positive lesions and over diagnostics are also increasing. Keep in mind the possible false positives of PET/CT and the results should be evaluated by clinicians according to the demographic, socio-cultural characteristics of countries, and the characteristics of endemic diseases in countries.

Keywords: FDG; PET-CT; False positity; Thoracic surgery

\section{Introduction}

PET/CT (Positron Emission Tomography) is one of the current methods for tumor detection. PET/CT provides a very effective biological point of view on oncologic imaging by introducing factors such as increased metabolism, protein synthesis and rapid cell proliferation in malignant cells through various pharmaceutical pathways. 18-fluoro deoxy glucose (FDG) is the most commonly used radio pharmacological agent in PET/CT imaging. The aim is to measure glucose metabolism within the body by FDG. It is a method that is applied with the idea that tumor cells use more glucose than normal cells. There are hundreds of studies that demonstrate the efficacy of PET/CT in terms of both diagnosis and treatment planning for thoracic lesions [1-3]. However, PET-CT has some false positives. False positive results of PET/CT may occur, especially in cases of inflammatory processes and macrophage dominance. This may lead to different diagnoses and unnecessary surgical interventions [4]. The aim of this study was to analysis surgically verified the false positivity of FDG PET-CT in our clinic between February 2014 and February 2019.

\section{Material and Methods}

Fifty-six patients with PET-CT positive results and preoperatively suspicious diagnosed as malignancy, but whose final 
histopathological results were reported as benign diseases were included in the study. Patients who had PET/CT due to intrathoracic mass and whose histopathological validation could be reached were included in the study. Large blood parameters, tumor markers were requested from all patients. Preoperative transthoracic fine needletrucut biopsy, fiberoptic bronchoscopy was performed. However, in these patients who could not be diagnosed with minimally invasive methods, the decision was made to operate in the multidisciplinary council. The patients were evaluated retrospectively according to age, gender, clinical parameters, PET-CT histopathological results and radiological images. Patients with type 2 diabetes mellitus, patients under 18 years of age and patients with missing data were excluded from our study. PET/CT was evaluated by nuclear medical experts. An average of 6-8 hours of fasting before the shooting, and in all cases, the blood glucose value was lower than $160 \mathrm{mg} / \mathrm{dl}$ before shooting, and images from the upper vertex to the middle of the thighs were taken.

F-18 FDG dose (8-15 mCi / kg) was calculated intravenously. In the semi-supine seats in the waiting room following the injection, images were taken approximately 45-60 minutes after the patients were allowed to rest at the appropriate room temperature. PET/ CT device (G.E. Discovery STE8), which is a combination of eight sections, $5 \mathrm{~mm}$ section thickness tomography unit and PET unit containing Bismuth Germenate block detector, was used for thorax images. The patients were vats and explored by thoracotomy, the lesions were excised. The operation was terminated after being reported as benign in the frozen section. In the definitive pathology, the real result of the benign event arose.

\section{Data Analysis}

A statistical analysis was performed using the Statistical Package for the Social Sciences program (SPSS, 20.0). Data were expressed as mean \pm SD. Frequencies and percentages were used for the categorical measures.

\section{Result}

Twenty of the patients (34\%) were female and 36 of them (66\%) male with the mean age $54,7 \pm 4,03$ (range 30-69) years. Wedge resection by posterolateral thoracotomy in 32 (57\%) patients, intraparenchymal punch biopsy by lateral thoracotomy in $10(18 \%)$ patients, wedge resection by VATS in $8(14 \%)$ patients, and intraparenchymal punch biopsy with VATS in $6(11 \%)$ patients was performed to reach a definitive diagnosis. No intraoperative or postoperative complications were observed in any patient. When the PET/CT values of all patients were examined, the mean SUV max value in all of the false positive cases was measured as 8,4 (0.626). In $21(38 \%)$ patients, the histopathological result was reported as tuberculosis and the mean SUVmax value was 5,1 (range 2,5-14). Other false positive results were sarcoidosis in $11(20 \%)$ patients with mean SUVmax value of 16,4 (range 14-26), Inflammation in nine $(16 \%)$ patients (pneumonia, abscess, BOOP etc.) with the mean SUVmax value of 10,2 (range2,2-25), intrapanimal lymph node in four (7\%)patients with the mean SUV max value of 7,9 (range 6,5-10,5), ruptured hydatid cyst in four (7\%) patients with the mean SUVmax value of 4,2 (range 2 to 5,9), hamartoma in four (7\%) patients with the mean SUVmax value of 1,2 (range $0,6-2,6)$ and lastly granulomatosis with polyangiitis in three $(5 \%)$ patients with the mean SUVmax value of 8,9 (range 7-10,8) was determined (Table 1, Chart 1 ). PET/CT images of cases for each diagnosis with SUVmax values was revealed in Figure 1,2,3,4,5,6,7 (Figure 1-7). There was no contradiction between the frozen and the definitive results in any cases. Patients with tuberculosis were referred to the treatment of antituberculous tuberculosis. The sarcoidosis patients were referred to the Chest Diseases clinic for follow-up and treatment. Patients with intrapaenchimal lymph node and hamartoma removal were followed up routinely from polyclinic every 3 months. Antibiotics were given according to the culture results of the patients who were reported as Inflammation (pneumonia, abscess). Albendazole 2x $400 \mathrm{mgr}$ per oral was started for 3 months in ruptured hydatid cysts. Patients with Granulomatosis with Polyangiitis were referred to the related internal clinics for treatment and follow up.

Table 1: False positive cases of PET CT.

\begin{tabular}{|c|c|c|}
\hline Pathological Diagnosis & n (56) & SUVmax (median) \\
\hline Tuberculosis & 21 & $5,1(2,5-14)$ \\
\hline Sarcoidosis & 11 & $16.4(14-26)$ \\
\hline Inflammation (pneumonia, abscess) & 9 & $10,2(2,2-25)$ \\
\hline Intraparenchymal lymph node & 4 & $7.9(6,5-10,5)$ \\
\hline Hydatid cyst (ruptured) & 4 & $4,2(2-5,9)$ \\
\hline Hamartoma & 4 & $1,2(0,6-2,6)$ \\
\hline Granulomatosis with Polyangiitis & 3 & $8,9(7-10,8)$ \\
\hline
\end{tabular}

SUVmax: Standardized maximum uptake value.

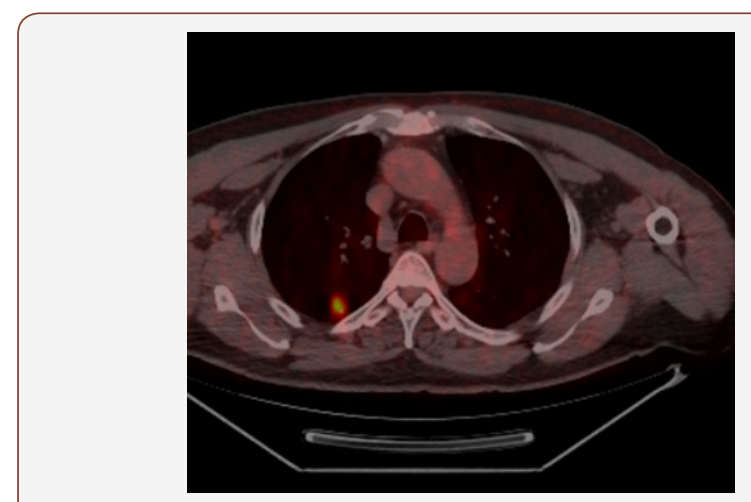

Figure 1: PET/CT image of the lesion located in the right upper lobe, SUVmax 7,3. The diagnosis was reported as tuberculosis.

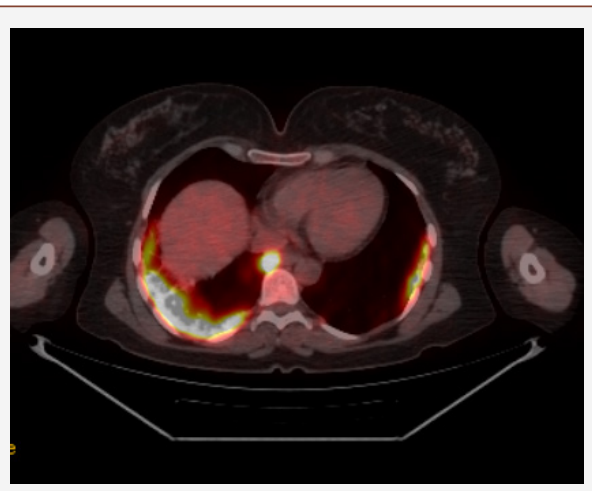

Figure 2: PET/CT image of the lesion located in bilateral lower lobes, SUVmax 26. The diagnosis was reported as sarcoidosis. 


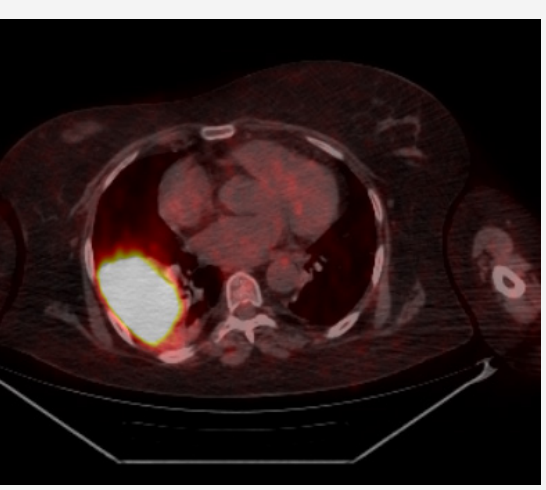

Figure 3: PET/CT image of the lesion located in the right lower lobe, SUVmax: 25. The diagnosis was reported as Bronchiolitis Obliterans with Organizing Pneumonia (BOOP).

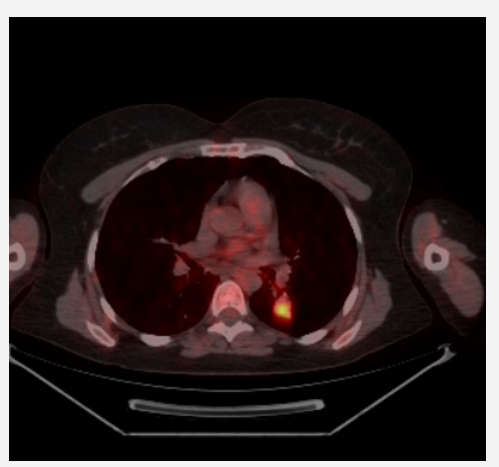

Figure 4: PET/CT image of the lesion located in the left lower lobe, SUVmax 7,1. The diagnosis was reported as intraparenchymal lymph node.

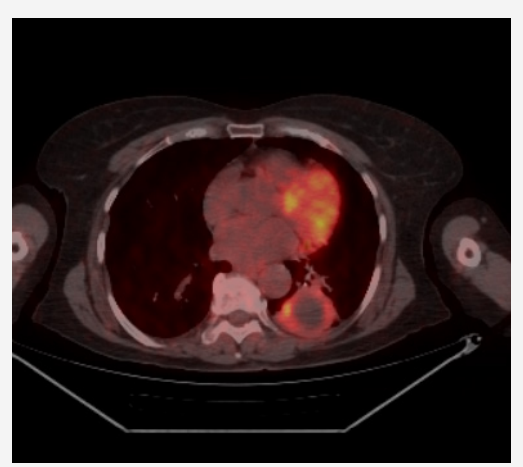

Figure 5: PET/CT image of the lesion located in the left lower lobe, SUVmax 5,9. The diagnosis was reported as hydatid cyst (ruptured).

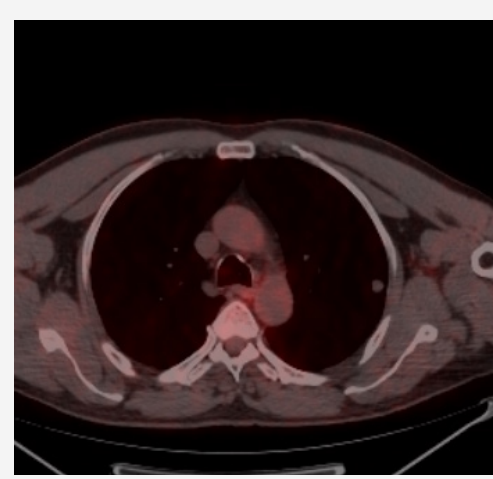

Figure 6: PET/CT image of the lesion located in the left lower lobe, SUVmax 1,2. The diagnosis was reported as hamartoma.

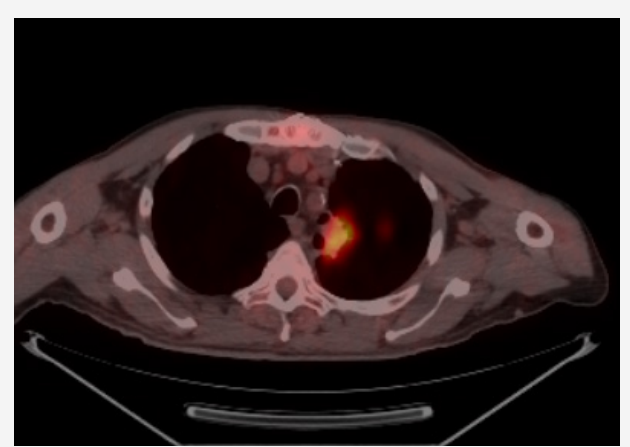

Figure 7: PET/CT image of the lesion located in the left upper lobe, SUVmax 10,8 . The diagnosis was reported as granulomatosis with polyangiitis.

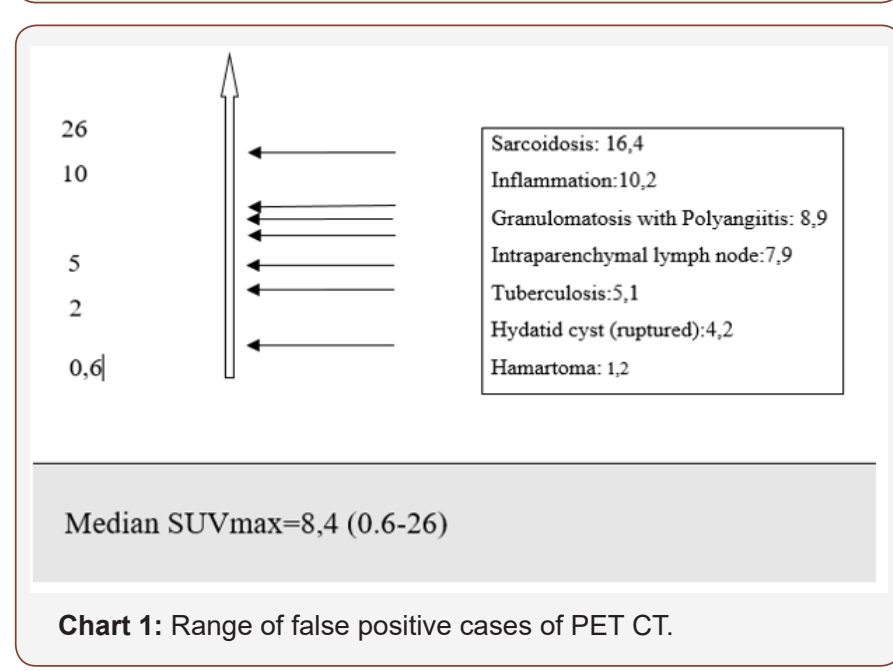

\section{Discussion}

Positron emission tomography is mainly used to distinguish benign lesions from malignant lesions, staging and follow-up to the response to chemotherapy. Some other pathologies (such as some benign tumors, inflammation and infection), which have increased glucose metabolism outside of malignant tumors, may show increased FDG uptake and sometimes even cause false positive results in oncologic PET/CT studies [5]. 18-fluoro deoxy glucose without a cancer-specific agent; shows increased involvement some inflammatory diseases such as sarcoidosis, tuberculosis, fungal infection and pulmonary abscess. However, unlike benign pathologies, FDG accumulation observed in malignant lesions until late is partly beneficial in the differential diagnosis. All infections and inflammations with active macrophages may cause positive involvement. This can lead to confusion in some cases [6]. In this study, false positives of PET/CT were demonstrated by surgical interventions. The literature on false positive or false negative results of PET-CT is limited. Takamochi [7]. reported the false positive rate as $14 \%$. Considering the increase in the use of PET/CT nowadays, it may be thought that this ratio may rise to a considerable extent. While PET/CT study was evaluated; false positive and false negative findings should be considered. Muscle tissue, brown fat tissue, thymus hyperplasia after chemotherapy, various physiological involvement, lung or mediastinal infection or inflammation and involvement due to non-malignant tumors lead to false positive results [8] The main basis of the study is to emphasize this situation. Also, surgical staples or suture lines following surgical 
resection can cause inflammatory or granulomatous changes with scar tissue formation with increased FDG uptake [9]. Radiotherapyrelated fibrosis may also lead to similar false positives [10]. There were no patients with a history of previous operations in the study. However, this situation should be considered when evaluating the PET/CT. Tuberculosis is still an important public health problem in developing countries. PET/CT study would not be useful in differentiating benign - malignant lesions with SUVmax value less than 2,5 in geographic regions where granulomatous diseases such as tuberculosis are common [11]. Tuberculosis is still important in our country. Tuberculosis was the most common cause of false positivity, in 21 (38\%) patients with the mean SUVmax value of 5,1 (range 2,5-14).

Sarcoidosis is a chronic multisystem noncaseating granulomatous disease. It is characterized by accumulation of $\mathrm{T}$ lymphocytes and mononuclear phagocytes, noncaseating epithelioid granulomas, and derangements of the normal tissue architecture in affected organs. The etiology is unknown but is though to be caused by an exaggerated cellular immune response. The disease has high FDG uptake up to aggressive malignant tumors [12]. Sarcoidosis in 11 patients with mean SUVmax value of 16,4 (range 14-26) was the second most common cause of false positivity in the study. In countries where hydatidosis is endemic, such as Turkey it can be difficult to distinguish of Hidatei cyst with lung cancer. Especially complicated cyst hydatid and other malignant lung diseases can be confused with their metabolic characteristics [13]. Four patients had false positivity due to ruptured hydatid cyst in the study, with the mean SUVmax value of 4,2 (range 2 to 5,9 ).

\section{Conclusion}

The use of PET/CT for thoracic lesions has been increasing over the last years. In addition, the detection of false positive lesions and over diagnostics are also increasing. It is aware of a possible false positivity and proper interpretation of the lesion can be achieved. For minimize possible false positives, PET/CT results should be evaluated by clinicians according to the demographic, socio-cultural characteristics of countries, and the characteristics of endemic diseases in countries. Also randomized controlled trials including larger case series are recommended about the topic.

\section{Acknowledgment}

None.

\section{Conflict of Interest}

No Conflict of Interest.

\section{References}

1. Detterbeck FC, Mazzone PJ, Naidich DP, Bach PB (2013) Screening for lung cancer: diagnosis and management of lung cancer, 3rd ed: American College of Chest Physicians evidence-based clinical practice guidelines Chest 143(5 Suppl): e78S-e92S.

2. Gould MK, Maclean CC, Kuschner WG, Rydzak CE, Owens DK (2001) Accuracy of positron emission tomography for diagnosis of pulmonary nodules and mass lesions: a meta-analysis JAMA 285(7): 914-924.

3. Ko Trukington TG, Coleman RE (2002) Clinical oncologic positron emission tomography: an introduction. Semin Roentgenol 37(2): 102109.

4. Shreve PD, Anzai Y, Wahl RL (1999) Pitfalls in oncologic diagnosis with FDG PET imaging: physiologic and benign variants. Radiographics 19(1): 61-77.

5. Mochizuki T, Tsukamoto E, Kuge Y, Kanegae K, Zhao S, et al. (2001) FDG uptake and glucose transporter subtype expressions in experimental tumor and inflammation models. J Nucl Med 42(10): 1551-1555.

6. Higashi T, Saga T, Nakamoto Y, Ishimori T, Mamede MH, et al. (2002) Relationship between retention index in dual-phase 18F-FDG PET, and hexokinase-II and glucose transporter-1 expression in pancreatic cancer. J Nucl Med 43(2): 173-180.

7. Takamochi K, Yoshida J, Murakami K, Niho S, Ishii G, et al. (2005) Pitfalls in lymph node staging with positron emission tomography in non-small cell lung cancer patients. Lung Cancer 47(2): 235-242.

8. Ichiya Y, Kuwabara Y, Sasaki M, Yoshida T, Akashi Y, et al. (1996) FDG-PET in infectious lesions: the detection and assessment of lesion activity. Ann Nucl Med 10(2): 185-191.

9. Shyn PB, Madan R, Wu C, Erturk SM, Silverman SG (2010) PET/CT pattern analysis for surgical staple line recurrence in patients with colorectal cancer. AJR Am J Roentgenol 194(2): 414-421.

10. Isles MG, McConkey C, Mehanna HM (2008) A systematic review and meta-analysis of the role of positron emission tomography in the followup of head and neck squamous cell carcinoma following radiotherapy or chemoradiotherapy. Clin Otolaryngol 33(3): 210-222.

11. Chen CJ, Lee BF, Yao WJ, Cheng L, Wu PS, et al. (2008) Dual-phase 18F-FDG PET in the diagnosis of pulmonary nodules with an initial standard uptake value less than 2.5. AJR Am J Roentgenol 191(2): 475479.

12. Guleria R, Jyothidasan A, Madan K, Mohan A, Kumar R, et al. (2014) Utility of FDG-PET-CT scanning in assessing the extent of disease activity and response to treatment in sarcoidosis. Lung India 31(4): 323-330.

13. Ozkan ZG, Turkmen C, Sanli Y, O Berker, S Tanju (2011) Accumulation of F-18 FDG in the infected pulmonary cyst in a patient with hydatid disease. Ann Nucl Med 25(6): 451-453. 